\title{
On the Radiation of Mesons
}

\section{with a Constant Transverse Momentum $P_{x}$ in Cosmic Ray Jets $\left(^{*}\right)$.}

\author{
G. Yekutieli
}

Department of Physics, The Weizmann Institute of Science - Rehovoth

(Nuovo Cimento, 13, $646(1959))$

ERRA'TA

CORRIGE

pag. 646, 2nd column:

5th line from top:

$$
n=\cos \varphi
$$$$
1 / n=\cos \varphi .
$$

5th line from bottom till the end of column:

In this case, we shall write

$$
-4 \pi \nu f(0)=k_{T}^{2},
$$

and

$$
\begin{gathered}
n^{2}=1-\frac{k_{T}^{2}}{k^{2}}=\cos ^{2} \varphi \text { or } p=\frac{P_{T}}{\sin \varphi}, \\
(p=\hbar k) ;
\end{gathered}
$$

In this case we shall write

$$
4 \pi v f(0)=k_{T}^{2},
$$

and for $k \gg k$

$$
\begin{gathered}
\frac{1}{n^{2}}=1-\frac{k_{2}^{2}}{k^{2}}=\cos ^{2} \varphi \text { or } p=\frac{P_{r}}{\sin \varphi}, \\
(p=\hbar k)
\end{gathered}
$$

